\title{
Automation of ISP 51 Spectrograph by NI LabVIEW and digital camera
}

\author{
D. Ghazaryan, T. Dadayan \\ National Instruments, Bagrevand 21/1, Yerevan, Armenia
}

E-mail: david.ghazaryan.no1@gmail.com

Received April 28, 2021

\begin{abstract}
In this project, the ISP 51 (Inductively Coupled Plasma) automation was implemented to perform digital calculations. For this, a digital camera was placed in the spectrograph detection area, and all the images obtained were processed using the LabVIEW software environment.
\end{abstract}

https://doi.org/10.52853/18291171-2021.14.2-99

Keywords: spectrograph, monochromator, spectral measurements, optical filter, intensity distribution, luminescence spectrum

\section{Introduction}

One of the methods of material properties is study of its spectral characteristics (transmission, absorption, reflection, and fluorescence). This is implemented by means of optical spectrometers. These devices consist of monochromator which splits light into spectral components and a measurement module which registers the light. Depending on the monochromator constructions the size of the spectrometers might vary from $10 \mathrm{~cm}$ to $1.5 \mathrm{~m}$. Both devices might have the same optical characteristics [1].

The light registration module of the spectrometers might also vary. In big UV-VIS-NIR spectrometers usually two photodiodes are used which are sensitive in the UV and VIS-NIR region of the light spectrum. In more compact fiber spectrometers, a CCD matrix is used to register the light. Usually, the wavelength range of these spectrometers is slightly narrower than the previous type of the spectrometers [2].

The optical spectrometers also are different by the method of the light delivery. In one kind of spectrometers the light travels in a free space while in the fiber spectrometers the light is delivered from/to the sample by means of optical fibers. Nowadays, fiber optic compact digital spectrometers are widely used, which allow measurements from ultraviolet to infrared spectral zones. The most known manufacturers of these devices are Ocean Optics, CNI lasers, Stellar Net, Thorlabs and others. These devices are compact, consisting of a diffraction grating and a CCD (Charge Coupled Device) matrix [3].

The advantage of the fiber spectrometers is that the measurement is carried out in few milliseconds and for all spectral range simultaneously meanwhile in large spectrometers the diffraction grating or the prism in the monochromator are rotating and each wavelength is measured individually thus the measurement takes longer time. Both types of the spectrometers are standing out for their high price (few thousands of USD), whilst the components necessary for developing a spectrometer are readily available in the laboratories [4].

In this article we develop a good alternative for both spectrometers taking their advantages (an optical resolution and simultaneous measurement for all spectral range). We use ISP 51 spectrograph and Canon sx110is digital camera for developing an optical spectrometer. The spectrograph splits light from an object into its component wavelengths so that it can be recorded 
and analyzed. It provides an image of defined bandwidth and wavelength. The ISP 51 is a threeprism spectrometer with two cameras with a focal length of 120 and $270 \mathrm{~mm}$. The device is designed to measure absorption, radiation and other spectral measurements. Break angles of prisms - 60 degrees, material - TF-1 sealant, operating range - 360-800 nm [5]. By means of LabVIEW we develop a program which analyzes the photos made by a CCD camera and perform wavelength calibration and spectrum analysis.

\section{Experimental part}

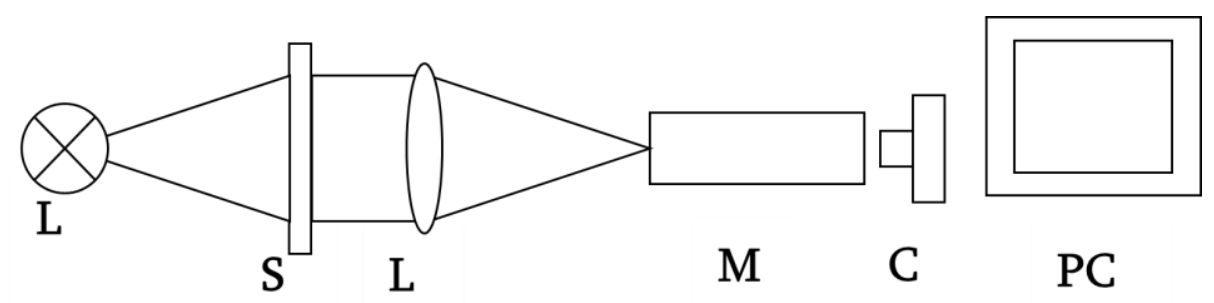

The ISP 51 monochromator and CANON IS 110 camera were used to measure the the penetration and reflection coefficients of optical samples. The diagram of the experimental device assembled to measure the sampling coefficient is shown in Fig. 1.

Fig. 1. Test device description: L-lamp, S-sample, L-lens, M-Monochromator, C-camera PC-Personal Computer.

The light passing through the sample is focused on the monochromator through the lens, in which the dissolution of the spectrum is recorded with the help of a camera. The spectrum picture is then processed on a computer by a corresponding program [6].

During the experiments, we used ordinary incandescent bulbs as a light source, and we used mercury bulbs to measure the device.

In our experiments, we used a Canon PowerShot 110 IS digital camera with 10x optical zoom and focal length adjustment. After removing the collimator, the digital camera is firmly installed at the output of the spectrograph. A collimated light source is placed in front of the entrance to the spectrograph. When measuring the emission spectrum, the sample is placed at the "spectrograph input" of the light source. The resulting device can now be called a spectrometer. For accurate instrument operation, it is necessary to measure its wavelength and transmission.

\section{Wavelength measurement}

To ensure the operation of the spectrometer, it must be calibrated so that the number of pixels of the CCD camera corresponds to a certain wavelength. [7] For this, a mercury lamp was used, which has spectral lines in a wide spectral range - Ultraviolet-visible. In particular, it has a pronounced radiation at wavelengths of $546,577,579 \mathrm{~nm}$. They are indicated by green and yellow lines, respectively. The last two spectral lines can also be used to estimate the optical resolution of the device.

Fig. 2a shows a photograph of the distribution of the spectrum and intensity of a mercury lamp by pixels: Fig. 2b. The image is black and white to determine the intensity of the pixels in grayscale numbers. The curve shown in Fig. 1b was generated using LabVIEW software by recording pixel values along the red line shown in Fig. 2a. To reduce the effect of noise on photographs on measurement accuracy, measurements taken from different parts of the photograph were averaged.

From Fig. 2 we see that the spectral lines at a distance of $2 \mathrm{~nm}$ are wonderfully different from 
each other. By measuring the distance between spectral lines in pixels, the pixels of a photo can be converted to wavelength. In particular, it turns out that $1 \mathrm{~nm}$ corresponds to 18 pixels. It should be noted that the intensity values do not have a unit of measurement, they depend on the recording time of the camera. The maximum value may be 255 , which corresponds to saturation.

a)

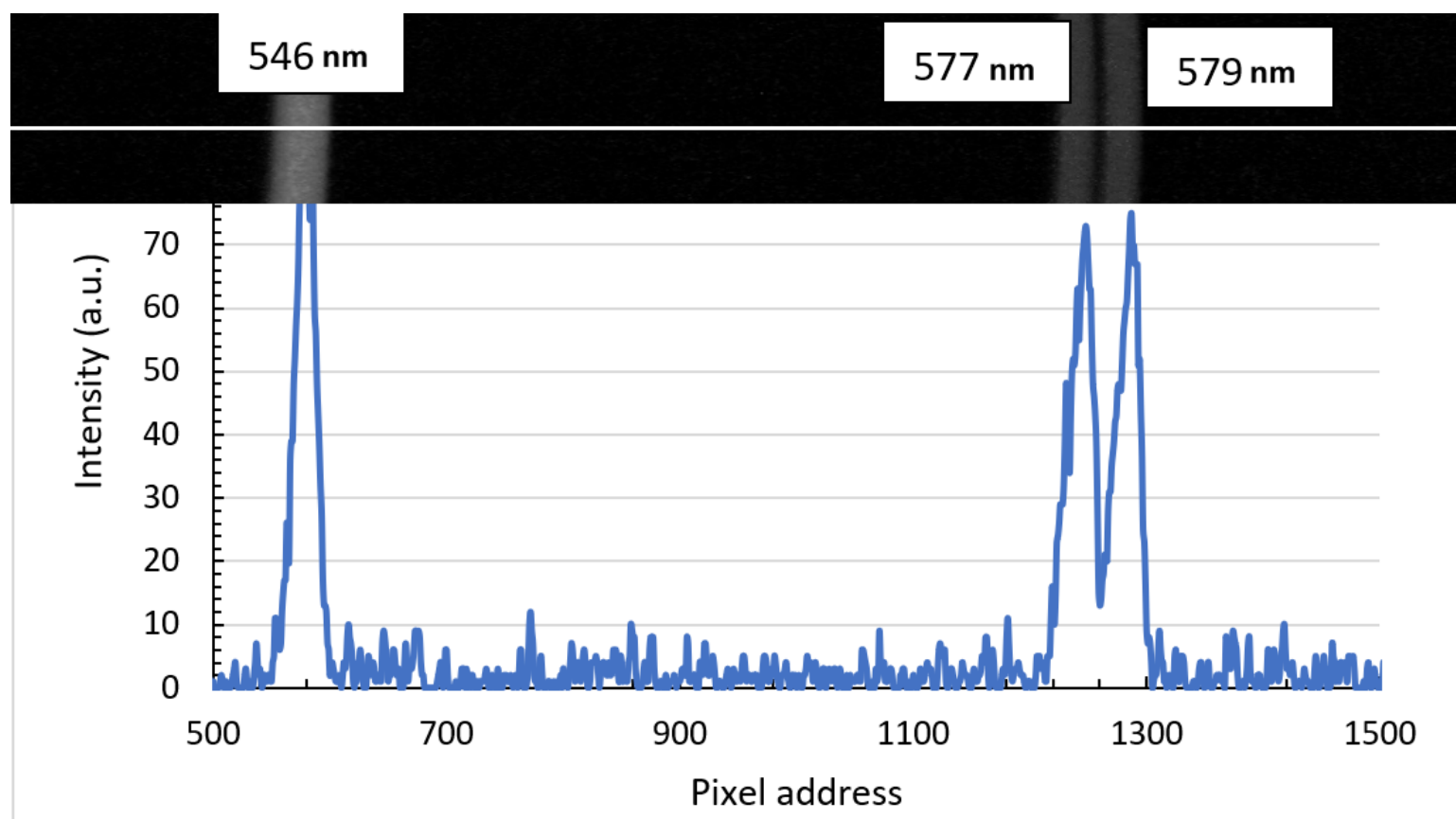

Fig. 2. a) Photo of the linear spectrum of a mercury lamp. b) Photo of pixel intensity distribution.

\section{Transmission}

The digital camera used has three color filters to create the final image. The absorption of these filters, red, green, blue, significantly affects the spectral composition of the incident light. Therefore, when recording the final image in black and white, this can lead to measurement error [8]. To avoid this, we also measured the gaps. Fixing the camera shutter speed, turning off color correction, all automatic functions were photographed by darkening the incandescent lamp spectrum with neutral filters. In Fig. 2 shows the spectrum of a mercury lamp, the spectrum of an incandescent lamp, attenuated by light filters NG7 and NG9. These filters are known for their nearly uniform absorption in the optical range.

The emission spectrum of light filters is determined by calculating the ratio of the light intensity of a given wavelength to the light intensity of an incandescent lamp at the same wavelength. In this calculation, the specified pixel intensity without light is subtracted from the numerator of the denominator [9].

In Fig.3, a drop in output power is noticeable at a wavelength of 2000-2200 nm, which corresponds to approximately $550 \mathrm{~nm}$ in the spectrum. This is caused by the absorption of the color filters installed on the camera.

For the NG-8 filter, we see that the transmission spectra match quite well. The differences for the HC-7 filter are obvious. This may be due to the camera's internal amplification mechanisms, which cannot be turned off. However, these data can be taken into account and to make corrections in future measurements 


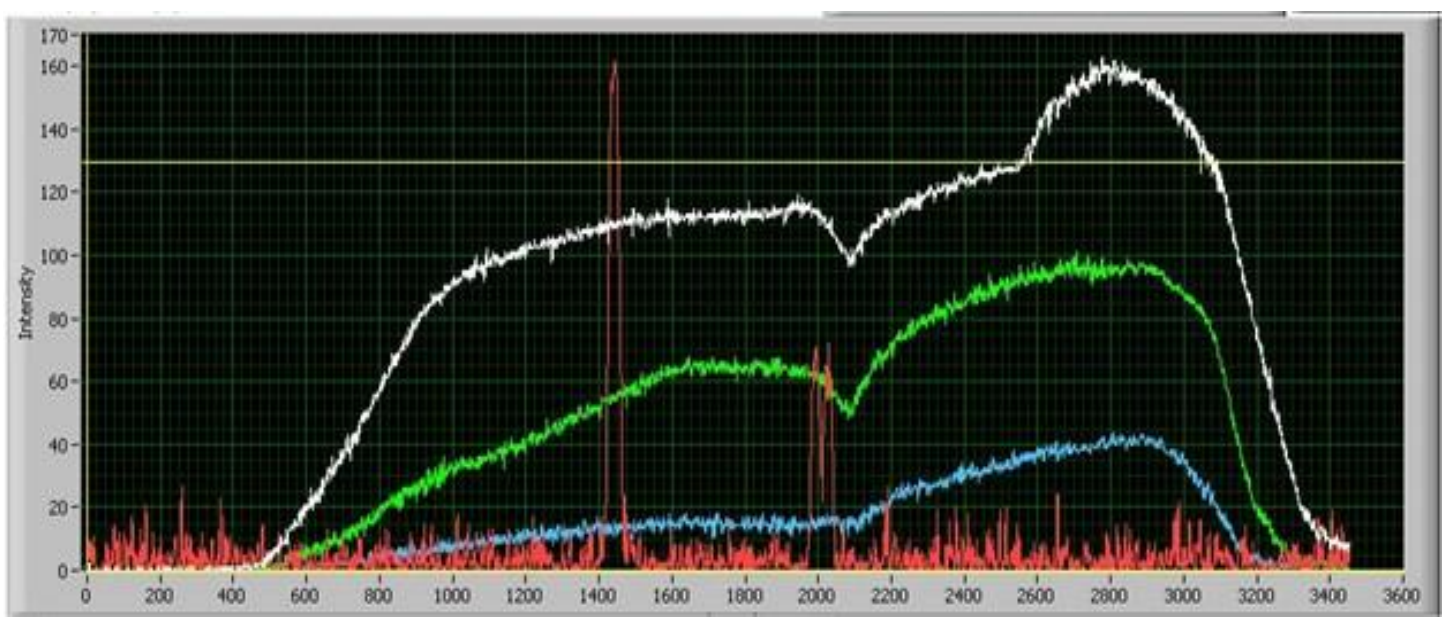

Fig. 3. The light spectrum is weakened by neutral filters (white curve), NG7 (green curve), NG8 (blue curve). The red curve corresponds to the spectrum of a mercury lamp.

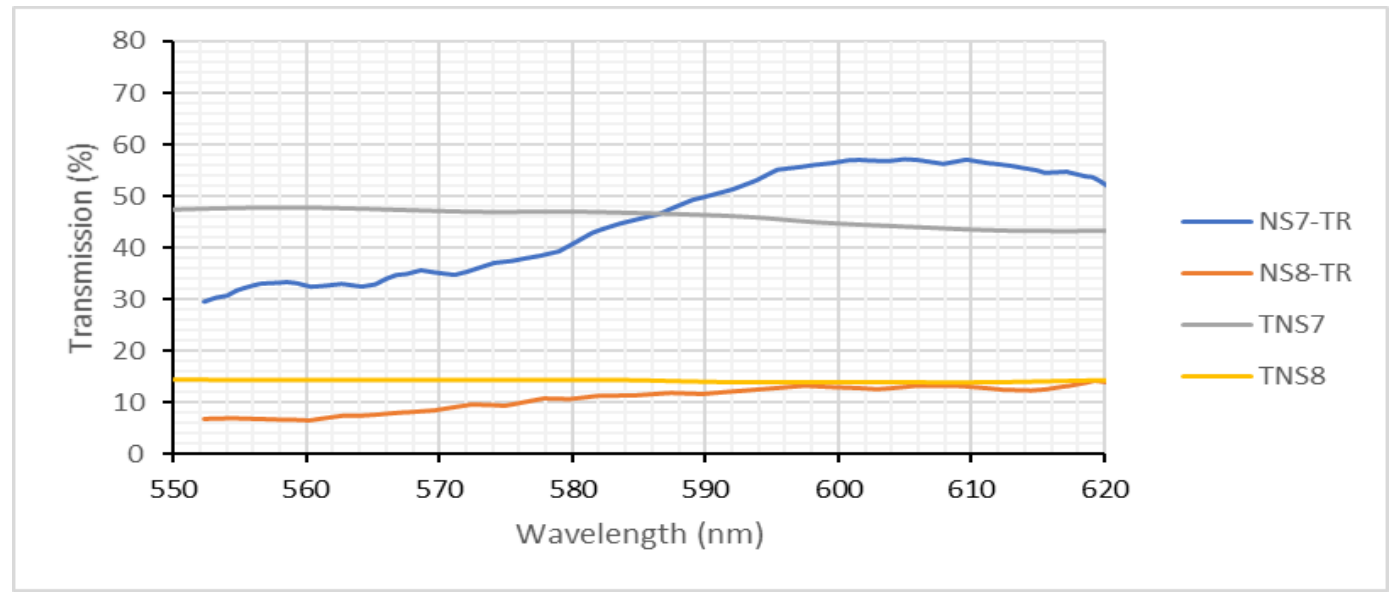

Fig. 4. Transmission spectra of light filters measured by our spectrometer (NG7-TR, NG8-TR) and commercial spectrometer (TNS7, TNS8).

\section{Software part}

To process the images, we created a program using the LabVIEW system-design platform and development environment for a visual programming language program that returns the numerical values of the intensities of the coordinates assigned to the given photo.

The block diagram of the mentioned program, which includes the line profile function. After photographing, the picture turns gray. The figure is presented in the form of a two-dimensional array, each of which corresponds to a number in the range 0-255. 255 is white, hence the highest intensity.

Thus, to measure the light intensity, the program outputs the coordinates of the area along which the bit values are measured, and then the dependence of the image brightness on the coordinate is plotted. 


\section{Device testing}

To test the device, the luminescence spectrum of the laser dye Rodamine $6 \mathrm{G}$ was recorded. This laser dye is known for its good alcohol solubility [10]. At a wavelength of $532 \mathrm{~nm}$, the dye emits luminescence in the range of 540-620 nm. Thus, this radiation can be recorded using a spectrometer that we have manufactured.

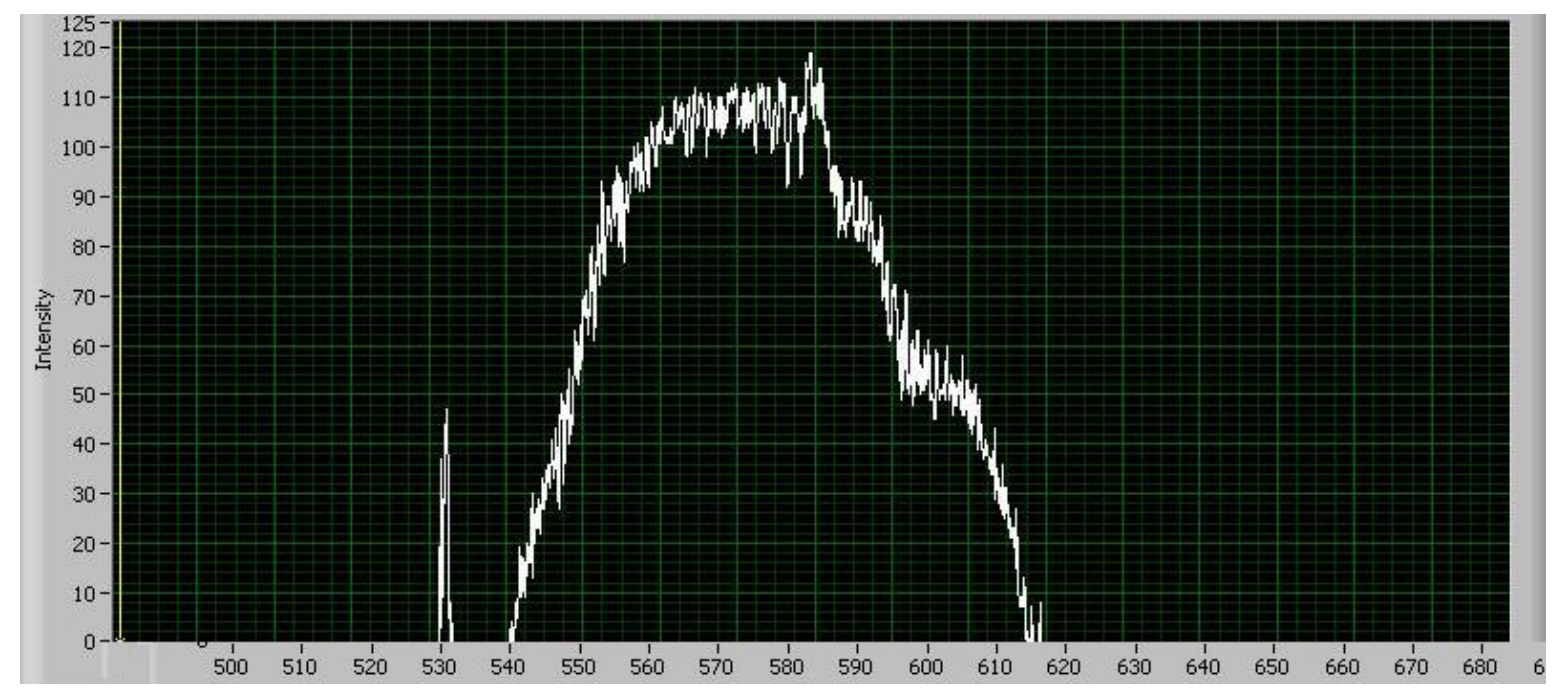

Fig. 6. The luminescence spectrum of Rodamine $6 \mathrm{G}$.

For this purpose, an alcoholic solution of Rodamine $6 \mathrm{G}$ at a concentration of 10-3 mol / 1 was prepared. The optical propulsion of the dye was realized by means of a $532 \mathrm{~nm}$ wavelength laser. The luminescence sector of the laser dye is shown in Fig. 6.

As we can see from the picture, the spectrometer recorded a radiation in the range of 540-620 nm. The peak recorded at $530 \mathrm{~nm}$ corresponds to the wavelength of the driving laser.

\section{Conclusions}

Thus, in the present work, automation of the ISP 51 spectrograph was carried out through the NI LabView and digital camera. The device was calibrated with a mercury lamp and neutral light filters. The resolution of the device is better than $2 \mathrm{~nm}$, which is quite a good indicator for the current compact spectrometers. The radiation spectrum of the laser dye was recorded by the device.

\section{References}

[1] M. Rigault, J. D. Neill, N. Blagorodnova, A. Dugas, M. Feeney, R. Walters, V. Brinnel, Y. Copin, C. Fremling, J. Nordin, J. Sollerman, Astronomy \& Physics 627 (2019) 22.

[2] I. Djermanov, S. Iordanova, I. Koleva, Second Intern. Workshop \& Summer School on Plasma Physics (2007).

[3] K. Fujii, Y. Endo, Y. Torigoe, S. Nakamura, T. Haruyama, K. Kasami, S. Miharab, K. Saitob, S. Sasakib, H. Tawara. Nuclear Instruments and Methods in Physics Research A 795 (2015) 293.

[4] B.L. Vasin, S.V. Malkova, M.V. Osipov, V.N. Puzyrev, A.T. Sahakyan, A.N. Starodub, S.I. Fedotov, A.A. Fronya, Technique for measuring the spectral sensitivity of the CCD array (RAN, P.N. Lebedev Physical Institute, Moscow, 2007). 
[5] G.S Landsberg. Optics (Science, Pleiades Publishing, 2006).

[6] E.I. Butikov. Optics (Nevskij Dialekt; BKHV-Peterburg, 2003).

[7] A. Alam, Z.A. Jaffery, Applications of Artificial Intelligence Techniques in Engineering 698 (2019) 333.

[8] A.V. Navid, R.M. Jeffrey, J.C. Parisa A. Bahrib, D. Parlevliet, Nannochloropsis sp. 8 (2015) 121.

[9] Y.V. Vizilter, S.Y. Jeltov, V.A. Knyaz, A.N. Xodarev, A.V. Morjin. Processing and analysis of digital images with examples on LabVIEW IMAQ Vision (M: DMK-Press, 2009).

[10] V.A. Donchenko, M.M. Zinoviev, A.A. Zemlyanov, V.A. Kharenkov, A.N. Panamaryova, $17^{\text {th }}$ Intern. Conf. of Young Specialists on Micro/Nanotechnologies and Electron Devices (2016) 370. 